\title{
La extensión de la obligatoriedad en el nivel secundario en países de América del Sur desde la perspectiva del derecho a la educación
}

\author{
Scioscioli, Sebastián \\ Centro de trabajo, Ciudad, País: Universidad de Buenos Aires -CONICET. Buenos Aires, Argentina \\ sebastianscioscioli@derecho.uba.ar
}

Lorente Rodríguez, Miriam

Centro de trabajo, Ciudad, País: Universidad Internacional de Valencia. Valencia, España

miriam.Iorente@campusviu.es

Ruiz, Guillermo Ramón
Centro de trabajo, Ciudad, País: Universidad de Buenos Aires-CONICET. Buenos Aires, Argentina
gruiz@derecho.uba.ar

\section{Resumen}

En América Latina muchas reformas educativas han avanzado en definiciones normativas sobre el derecho a la educación con objetivos muy diversos tales como la mejora de la calidad educativa, o bien la emancipación de los sectores oprimidos de las sociedades. También podría considerarse que dichas reformas obedecen al impacto del derecho internacional de los derechos humanos al interior de los derechos nacionales. Así, se presentó a la ampliación de los años de educación escolar obligatoria como una política que forma parte del contenido del derecho a la educación. En consecuencia, en algunos países esta política ha fijado un nuevo rango de estudios obligatorios que incluye toda la educación secundaria. Entre los argumentos esgrimidos por las autoridades se encuentran la atención a las demandas educativas de los sectores que no se encontraban escolarizados y también se sostiene que con ello se favorecería el logro de una ciudadanía más efectiva en las jóvenes generaciones, así como una formación para el trabajo que no descuide la función propedéutica de la escolarización secundaria de modo de garantizar el acceso a los estudios superiores.

En esta presentación nos concentraremos en los conceptos de derecho a la educación y de obligatoriedad escolar para adentrarnos en la revisión de algunas de las reformas recientes de cinco países de América del Sur que han establecido la obligatoriedad de la escuela secundaria. En segundo lugar, se analizan las políticas educativas que consideran a la educación obligatoria como objeto de análisis desde un enfoque de derechos. Cuestiones como la obligatoriedad y la transferencia de políticas y discursos entre los países son discutidas. Aquí la comparación transnacional se focaliza en las políticas de reforma de nivel secundario a la luz del enfoque de derecho humanos; se tendrá en cuenta la evolución de la cobertura y el rendimiento interno de los países estudiados. En términos metodológicos el diseño de esta investigación incluye técnicas cuantitativas, como el análisis de estadísticas educativas regionales, y cualitativas, tales como el análisis de contenido de documentos normativos provenientes de los países considerados.

\section{Abstract}

The Latin American educational reforms have made normative definitions regarding the right to education (in several directions and with diverse objectives). In this paper we discuss the topics of right to education and compulsory schooling since high secondary education was made compulsory because of these reforms in some South American countries. We analyze the changes which have taken place in secondary education in four countries that are members of the Southern Common Market (MERCOSUR): Argentina, Brazil, Paraguay and Uruguay. I also we take into account one country which is located in same region: Bolivia. Thus, we track the recent trajectory of government policies for secondary education, which have been modified by these five South American countries. We discuss the meaning of secondary education for all, and the issues that are involved, from a regional perspective in order to identify current convergences in academic organizations of secondary school and the constraints of their implementations in accordance with academic traditions and the educational local settings.

Palabras clave: derecho a la educación, escuela secundaria, reformas educativas, políticas de transferencia, América del Sur.

Keywords: right to education, secondary school, educational reforms, transfer policies, South America. 


\section{INTRODUCCIÓN}

El derecho a la educación y la obligatoriedad escolar constituyen términos (para las ciencias jurídicas y las ciencias de la educación) que suelen ser vinculados en diferentes ámbitos (políticos y académicos). De esta forma se comprenden algunas razones por las cuales muchas reformas escolares han avanzado en definiciones normativas sobre el derecho a la educación como premisa para la superación de desigualdades sociales. En este sentido, la extensión de la obligatoriedad escolar hasta completar toda la educación secundaria ha sido una de las políticas que algunos Estados latinoamericanos han llevado adelante durante las últimas tres décadas como forma de atender demandas educativas y así favorecer el logro de una ciudadanía más efectiva y la promoción de una educación democratizadora. En esta comunicación nos concentraremos en la revisión de algunas de las reformas recientes de cinco países de América del Sur que han establecido la obligatoriedad de la escuela secundaria como forma de garantizar el goce del derecho a la educación.

\section{DEFINICIONES CONCEPTUALES: LA EDUCACIÓN COMO DERECHO FUNDAMENTAL}

Una manera de realizar la elucidación de este concepto proviene del derecho internacional de los derechos humanos. En este encuadre el derecho a la educación comprende desde el plexo normativo internacional (y también local) un importante catálogo de obligaciones a cargo de los Estados nacionales. Estas obligaciones, en función del contenido de este derecho, pueden clasificarse como (Ruiz y Scioscioli,2017):

1. Obligación de respetar. Esta obligación se observa cuando el derecho a la educación se comporta justamente como derecho de prestación negativa o no-intervención. La obligación de respetar implica que el Estado se abstenga de injerir en el disfrute del derecho social.

2. Obligación de proteger. Esta obligación exige a los Estados adoptar leyes u otras medidas que resulten necesarias para evitar o prevenir que los particulares (u otros sujetos diferentes al Estado como, por ejemplo, particulares o sea el sector privado) produzcan dichos perjuicios.

3. Obligación de cumplir, realizar, y garantizar. Este aglomerado de obligaciones es el más interesante en función del contenido social del derecho a la educación y de cara al contexto de exclusión social que afecta a los países aquí estudiados. Esta obligación se define como a la necesidad de que los Estados reconozcan este derecho en sus ordenamientos jurídicos y que adopten una política nacional acompañada de un plan detallado para el ejercicio del derecho. Como puede desprenderse de este somero análisis, las obligaciones internacionales en materia de derechos humanos pueden traducirse en un catálogo de contenidos básicos de tales derechos. La obligatoriedad escolar secundaria, entendida a priori como la extensión de los estudios generales de nivel post-primario y graduales, accesible y válida para todas las personas involucra a todos los aspectos que estructuran el contenido del derecho a la educación como derecho humano y redefinen también el piso de las obligaciones de los Estados nacionales para con este derecho. El contexto latinoamericano tiene mucho que aportar dadas las recientes reformas normativas que en los países de la región existieron en materia educativa y que apuntaron a reconocer las condiciones materiales de vigencia del derecho a la educación en sus territorios y que culminaron en la adopción de incluso nuevas cláusulas constitucionales que reconocieran la legitimidad del reclamo por una concepción de la educación como derecho social y por una mayor interpelación hacia el Estado a efectos de que éste tenga un rol más activo como garante de los contenidos presentes en dicho derecho (Gargarella y Courtis,2009).

\section{LAS REFORMAS EN LA ESCUELA SECUNDARIA EN CINCO PAÍSES}

Para analizar la situación educativa de los países de América del Sur se debe considerar que sus desarrollos educativos reflejan las características diversas de sus respectivas estructuras sociales. Las reformas contemporáneas se asientan sobre sistemas escolares muy distintos en lo que atañe a su organización institucional y académica. En el siguiente cuadro se resume un panorama de la educación escolar de cinco países de América del Sur en función de sus regímenes de gobierno y características demográficas. 
Tabla 1: Organización institucional y estructura académica de los sistemas escolares de cinco países de América del Sur.

\begin{tabular}{|c|c|c|c|c|c|}
\hline $\begin{array}{c}\text { País } \\
\text { Indicador }\end{array}$ & Argentina & Bolivia & Brasil & Paraguay & Uruguay \\
\hline $\begin{array}{l}\text { Régimen de } \\
\text { gobierno }\end{array}$ & Federal & $\begin{array}{l}\text { Régimen centralizado } \\
\text { con niveles de } \\
\text { descentralización y } \\
\text { autonomías } \\
\text { (Estado plurinacional) }\end{array}$ & Federal & $\begin{array}{c}\text { Régimen } \\
\text { centralizado }\end{array}$ & $\begin{array}{c}\text { Régimen } \\
\text { centralizado }\end{array}$ \\
\hline $\begin{array}{l}\text { Organización } \\
\text { política }\end{array}$ & $\begin{array}{l}23 \text { Provincias y } \\
1 \text { Ciudad } \\
\text { Autónoma }\end{array}$ & 9 Departamentos & $\begin{array}{l}26 \text { Estados, } \\
5.564 \\
\text { Municipios y el } \\
\text { Distrito Federal }\end{array}$ & 17 Departamentos & 19 Departamentos \\
\hline Población & 43.4 millones & 10.72 millones & 207.8 millones & 6.7 millones & 3,4 millones \\
\hline Pob. escolar & 13.004 .334 & 3.005 .841 & 60.693 .813 & 2.278 .803 & 990.998 \\
\hline $\begin{array}{c}\text { Rango de } \\
\text { obligatoriedad }\end{array}$ & 14 años & 14 años & 14 años & 14 años & 14 años \\
\hline $\begin{array}{l}\text { Reforma } \\
\text { educativa }\end{array}$ & $\begin{array}{l}\text { Año } 2006 \text { Ley } \\
\text { de Educación } \\
\text { Nacional } \\
\text { N. }{ }^{\circ} 26.206\end{array}$ & $\begin{array}{c}\text { Año } 2010 \text { Ley de la } \\
\text { Educación Avelino } \\
\text { Siñani-Elizardo Pérez } \\
\text { N. }{ }^{\circ} 70\end{array}$ & $\begin{array}{c}\text { Año } 1996 \text { Ley } \\
\text { de Directrices y } \\
\text { Bases de la Ed. } \\
\text { Nacional } \\
\text { N. }{ }^{\circ} 9.394\end{array}$ & $\begin{array}{l}\text { Año } 1998 \text { Ley } \\
\text { General de } \\
\text { Educación } \\
\text { N. }{ }^{01.264}\end{array}$ & $\begin{array}{c}\text { Año } 2008 \text { Ley } \\
\text { de Educación } \\
\text { N. }{ }^{\circ} 18.437\end{array}$ \\
\hline
\end{tabular}

Fuente: Elaboración propia a partir de las normativas educativas y datos demográficos de cada país.

\section{ALGUNOS INDICADORES EN RELACIÓN CON LOS LOGROS EDUCATIVOS}

La evolución y expansión del derecho a la educación ha permitido plantearse la finalización del nivel secundario de educación como una meta y, en consecuencia, su obligatoriedad como un deber de los Estados en cumplimiento con la agenda política en educación. Todo ello respaldado por la prolija bibliografía que avala los numerosos beneficios de la educación, en general y de la educación secundaria, en particular (Caillods y Hutchinson, 2001; Tedesco, 2012; Vila, 2003). Ello también constituye la premisa fundante que se encuentra en la base las Agendas Mundiales de Educación (en lo que a América Latina concierne se pueden mencionar las denominadas Metas Educativas 2021 y la Agenda de Educación, ODS 2030)1.

\footnotetext{
1 Diversos Organismos Internacionales como la Organización de Estados Iberoamericanos -OEI- (2010, 2016) o la Comisión Económica para América Latina y el Caribe -CEPAL- (1997; 2010a; 2010b) coinciden en señalar que son doce el mínimo de años de escolarización necesarios para evitar caer en la pobreza y garantizar el futuro laboral y social de los jóvenes, lo que coincide con la finalización de la educación secundaria superior/completa. Igualmente, el Sistema de Información de Tendencias Educativas en América Latina -SITEAL(2010) señala que el acceso y la permanencia de los niños y las niñas en el sistema educativo durante al menos 12 años son condiciones necesarias para conseguir universalizar el acceso al conocimiento y una educación de calidad.
} 
Tabla 2: Tasas netas de acceso a la educación primaria y a la educación secundaria, según país y año. Cinco países de América del Sur.

\begin{tabular}{|c|c|c|c|c|}
\hline \multirow{2}{*}{$\begin{array}{c}\text { Indicador } \\
\text { País }\end{array}$} & \multicolumn{4}{|c|}{ ACCESO } \\
\cline { 2 - 5 } & 2007 & $2015^{1}$ & 2007 & $2015^{2}$ \\
\cline { 2 - 5 } & 99,1 & 99,4 & 79,2 & 88,5 \\
\hline Argentina & 95,4 & 89,8 & 73,5 & 78 \\
\hline Bolivia & 91,6 & 92,7 & 73,2 & 81,3 \\
\hline Brasil & 91,3 & 88,5 & 58,8 & 66,5 \\
\hline Paraguay & 97,8 & 97,2 & 67,6 & 81,3 \\
\hline Uruguay & \multicolumn{4}{|c|}{ Tasa neta de matrícula en secundaria } \\
\hline
\end{tabular}

Fuente: Elaboración propia a partir de la bases de datos del Banco Mundial, de la CEPAL y del SITEAL.

Notas:

${ }^{1}$ Los datos de Bolivia pertenecen a 2016 y los de Paraguay a2012.

${ }^{2}$ Los datos de Bolivia pertenecen a 2016 y los de Paraguay a2012.

Tabla 3: Tasas de eficiencia interna y permanencia en la educación secundaria para el último año disponible, según país y año. Cinco países de América del Sur.

\begin{tabular}{|c|c|c|}
\hline \multirow{3}{*}{$\begin{array}{l}\text { Indicador } \\
\text { País }\end{array}$} & \multicolumn{2}{|c|}{ EFICIENCIA INTERNA Y PERMANENCIA } \\
\hline & $\begin{array}{c}\text { Alumnos con dos o más años de retraso } \\
\text { escolar en secundaria }(\%)\end{array}$ & $\begin{array}{c}\text { Tasa de Jóvenes fuera de la escuela en edad de } \\
\text { asistir al nivel secundario superior }\end{array}$ \\
\hline & $2015^{1}$ & 2013 \\
\hline Argentina & 32,6 & 11,4 \\
\hline Bolivia & 15,5 & 19,6 \\
\hline Brasil & 26,6 & 16,7 \\
\hline Paraguay & 18,5 & 30,5 \\
\hline Uruguay & 30,2 & 18,4 \\
\hline
\end{tabular}

Fuente: Elaboración propia a partir de la bases de datos del Banco Mundial, de la CEPAL y del SITEAL. Nota:

${ }^{1}$ Los datos de Brasil y Uruguay pertenecen a 2015; los de Argentina, Bolivia y Paraguay a 2014. 
Tabla 4: Jóvenes y adultos con nivel primerio y nivel secundario completo, según país y año. Cinco países de América del Sur.

\begin{tabular}{|c|c|c|}
\hline \multirow{2}{*}{$\begin{array}{c}\text { Indicador } \\
\text { País }\end{array}$} & $\begin{array}{c}|c| \\
\text { Personas de 15 a 19 años con primaria } \\
\text { completa (\%) }\end{array}$ & $\begin{array}{c}\text { Personas de 20 a 24 años con secundaria } \\
\text { completa (\%) }\end{array}$ \\
\cline { 2 - 4 } & 2014 & 2014 \\
\hline Argentina1 & 98.4 & 65.6 \\
\hline Bolivia2 & 95.3 & 65.6 \\
\hline Brasil & 96.2 & 79.1 \\
\hline Paraguay & 93.7 & 61.9 \\
\hline Uruguay & 97.9 & 41.6 \\
\hline
\end{tabular}

Fuente: Elaboración propia a partir de la bases de datos del Banco Mundial, de la CEPAL y del SITEAL.

Notas: ${ }^{1}$ Los datos de conclusión de la educación primaria y secundaria pertenecen al área urbana.

${ }^{2}$ Los datos de pertenecen a 2013.

En lo que concierne a las Tablas 2, 3 y 4 puede apreciarse que la educación secundaria muestra un panorama aún más complejo que la educación primaria, con una región cuyos países caminan a distintas velocidades hacia la conclusión del nivel. El acceso al nivel de educación secundaria y su conclusión distan mucho de las alentadoras cifras que ofrece la educación primaria en los países de América del Sur aquí analizados. En este nivel educativo la variabilidad y dispersión de los datos aumenta considerablemente en los resultados de los distintos países, pero sobre todo en los datos desagregados en función del nivel de ingresos y el área geográfica de residencia.

La tasa neta de matrícula en secundaria es bastante inferior que la del nivel primario, aun cuando la secundaria, en su totalidad, forma parte de la enseñanza obligatoria, lo que da buena cuenta de los elevados niveles de fracaso y abandono escolar y, en cierta medida, de la incapacidad del sistema educativo y de las políticas estatales para retener a los estudiantes hasta el último grado de lasecundaria.

Así pues, podemos observar que en primaria el acceso se encuentra prácticamente garantizado en los países del cono sur analizados, con una ligera reducción en países como Bolivia y Paraguay. Sin embargo, el acceso al nivel secundario se reduce en torno a un promedio del $10 \%$ en países como Argentina, Bolivia y Brasil en comparación con el nivel primario y en torno al $20 \%$ en Paraguay y Uruguay. En estos dos últimos países los problemas de retención escolar y el rezago educativo se hacen más patentes. La comparación a través de los años permite destacar, en mayor medida, el esfuerzo realizado por Argentina y Uruguay, donde la tasa neta de acceso a secundaria ha aumentado en torno a un $10 \%$ promedio en el presente, con respecto al año 2007.

Si se considera exclusivamente el nivel secundario de educación, tal y como fue advertido, la dispersión de los datos entre países se hace más patente con respecto al nivel primario de educación. En términos de permanencia y eficacia interna esta situación puede ser advertida, si bien es cierto, la tendencia general es la de unos niveles bastante elevados de rezago escolar. De hecho, en torno a 3 de cada 10 alumnos que asisten, a la educación secundaria, han repetido curso en 2 o más ocasiones y dos de cada 10 en Bolivia y Paraguay. En países como Argentina y Brasil, el rezago existente afecta en menor medida a la permanencia escolar. Mayor incidencia tiene en países como Bolivia y Uruguay. La situación más alarmante se da en Paraguay, donde 3 de cada 
10 alumnos en edad de asistir a la educación secundaria superior, no lo hacen. No obstante, este último indicador refleja no sólo el abandono escolar que se produce en dicha etapa educativa, sino también aquellos que ni siquiera llegaron a acceder al mismo.

Igualmente, las cifras se vuelven mucho más desalentadoras al observar la conclusión de la educación secundaria en todos los países del cono sur que son analizados en este estudio. Sólo en Brasil la situación se revela más alentadora, dado que 8 de cada 10 alumnos consiguen concluirla. En la educación primaria, sin embargo, la conclusión del nivel está prácticamente universalizada. Si se analiza la conclusión de ambos niveles combinados, podemos identificar diversos perfiles de países. El primero de ellos sería el de países con un alto egreso en el nivel primario y secundario, el caso de Brasil. En segundo lugar, países como Argentina y Bolivia con un egreso alto en primaria y un egreso medio en secundaria. Finalmente, el tercer perfil sería el de países con alto egreso en el nivel primario, pero bajo en el nivel secundario. Es el caso de Paraguay y, sobre todo, de Uruguay. En estos últimos países sólo 6 y 4 alumnos, respectivamente, consiguen culminar la educación secundaria seis años después de la edad de finalización teórica de la misma. Esta situación es alarmante, eminentemente, en el caso uruguayo. A este respecto, Aristimuño (2009), basándose en los estudios realizados por ANEP/TEMS (2000 y 2003) concluye que «el conjunto de expectativas, creencias y relaciones sociales que rodean al joven, comenzando por su familia y siguiendo por su grupo de pares, son cruciales en el momento de tomar la decisión de desafiliación. También es relevante una vez más la historia escolar, específicamente la condición de repetidor»(p.189).

\section{DISCUSIÓN Y CONCLUSIONES}

En esta comunicación la comparación transnacional se ha focalizado en las políticas de reforma de nivel secundario a la luz del enfoque de derechos humanos y tomó el concepto de derecho a la educación como dimensión de análisis principal para analizar los procesos de reformas que algunos países de América del Sur han llevado adelante. Ahora bien, consideramos necesario destacar algunas precisiones conceptuales. Las posturas recientes han reconocido la existencia no sólo de una educación comparada sino de diversas educaciones comparadas (Ruiz y Acosta, 2016). Los críticos dentro del campo incluso han distinguido una educación comparada académica (que intenta dar explicaciones a través de la indagación cross-nacional) de una educación comparada intervencionista (que orienta al desarrollo y reformas de los sistemas educativos). Otros han discutido la política y la economía de la comparación, esto es las estrategias y los estilos fluctuantes de los estudios cross-nacionales implicados por los cambios devenidos de los intereses y constelaciones políticas y económicas en general y bajo las condiciones de un contexto globalizante en particular (Steiner-Khamsi, 2010). Desde la perspectiva panorámica del análisis socio-históricos, estos autores y otros autores sostienen que la comparación ha pasado de ser un enfoque basado en una teoría relacionada con un enfoque analítico a uno basado en una herramienta política de gobernanza (Nóvoa y Yariv-Mashal, 2003). Finalmente, durante las últimas tres a cuatro décadas se ha desarrollado una serie de estudios de lógica global que ya no se concentran sobre instituciones educativas de distintos Estados nacionales, o sociedades, o culturas sino sobre los procesos y las transformaciones educativas inherentemente trasnacionales y que tendrían un carácter sistémico mundial (Schriewer, 2014). En esta revisión de los supuestos epistemológicos de la disciplina, uno de los temas históricos de la educación comparada, la transferencia de prácticas, políticas y supuestos teóricos, aparece también puesto bajo análisis. Más que considerar la transferencia un proceso lineal y unidireccional, cabría pensarla como circular y, en cierta forma, como un proceso recíproco. Este concepto resulta particularmente útil para estudiar las reformas educativas, ya que permite entender cómo estas avanzan y retroceden entre diferentes países y dentro de ellos, sobre todo en casos cuya organización institucional es federal, extensa y compleja (como Argentina o Brasil). Aunque este ejercicio intelectual referido a la transferencia de políticas o discursos de reformas también es útil en países de estructura territorial unitaria (como Bolivia, Paraguay y Uruguay) dado que la aplicación de políticas y reformas trasvasa algunas de las características de los regímenes de gobierno. Las nuevas realidades espaciales mantienen, pero también desbordan al Estado-nación como eje analítico central para el estudio de la transferencia. Las dimensiones espaciales en las cuales operan las instituciones escolares, los sistemas y las políticas educativas revelan nuevos modos en los que se gobierna la educación formal y su organización en contextos geográficos con múltiples movilidades, migra- 
ciones y minorías educativas. Un primer acercamiento a este análisis puede estar dado por uno de los conceptos que más ha sido invocado en los procesos de reforma de la escuela secundaria en la región (sobre todo cuando se torna obligatoria): el derecho a la educación.

Ahora bien, en nuestro análisis en particular, nos preguntamos si la extensión de la obligatoriedad al nivel secundario de los sistemas escolares -como parte de la promoción del derecho a la educaciónse puede interpretar como un reflejo de estos procesos de transferencia internacional y si ello guarda relación con las implicancias efectivas que el derecho a la educación posee como derecho humano fundamental. Vale destacar que en este estudio hemos intentado introducir algunos cuestionamientos que permitan interpretar las lógicas de las reformas escolares y, eventualmente en el futuro, vislumbrar la eficacia de las políticas públicas derivadas en este terreno.

\section{REFERENCIAS BIBLIOGRÁFICAS}

Aristimuño, A. (2009). El abandono de los estudios del nivel medio en Uruguay: Un problema complejo y persistente. Revista Iberoamericana sobre Calidad, Eficacia y Cambio en Educación, (7), 4, 180-197. Recuperado 29/04/2018 de http:// www.redalyc.org/articulo.oa?id=55114094010.

Banco Mundial (2018). DataBank. Recuperado 20/04/2018 de http://databank.worldbank.org/data/home.aspx.

Caillods, F. y Hutchinson, F. (2001). ¿Aumentar la participación en la educación secundaria en América Latina? Diversificación y equidad. En C. Braslavsky (Orq.), La educación secundaria. ¿Cambio o inmutabilidad? Análisis y debate de procesos europeos y latinoamericanos contemporáneos (pp. 21-64). Buenos Aires: Ediciones Santillana.

CEPAL (1997). Panorama social de América Latina 1997. Santiago de Chile: NacionesUnidas.

(2010a). Panorama social de América Latina 2010. Santiago de Chile: CEPAL.

(2010b). El progreso de América Latina y el Caribe hacia los Objetivos de Desarrollo del Milenio. Desafíos para lograrlos con igualdad. Santiago de Chile: Naciones Unidas.

(2018). CEPALSTAT. Base de datos y publicaciones estadísticas. Recuperado 20/04/2018 de http://estadisticas.cepal.org/ cepalstat/Portada.html.

Gargarella, R. y Courtis, C. (2009). El nuevo constitucionalismo latinoamericano: promesas e interrogantes. CEPAL - Series Políticas Sociales, N. ${ }^{\circ} 53$.

Nòvoa, A. y Yariv-Mashal, T. (2003). Comparative research in education: A mode of governance or a historical journey? Comparative Education, 39 (1),423-438.

OEI (2010). Metas educativas 2021.La educación que queremos para la generación de los bicentenarios. Documento final. Madrid:OEI.

(2016): Miradas sobre la Educación 2016. Avance en las Metas Educativas 2021. Madrid: OEl.

Ruiz, G. y Acosta, F. (2016). Repensando la educación comparada: lecturas desde Iberoamérica. Entre los viajeros del siglo XIX y la globalización. Barcelona:Octaedro.

Ruiz, G. y Scioscioli, S. (mayo de 2017). El derecho a la educación y la educación secundaria obligatoria en países de América Latina: algunos casos nacionales. Disertación presentada en el III Encuentro de Educación Internacional y Comparada Innovación en educación, instituciones y actores en perspectiva internacional y comparada. Ciudad de México, México.

Schriewer, J. (2014). Neither orthodoxy nor randomness: differing logics of conducting comparative and international studies in education. Comparative Education 50 (1),84-101.

SITEAL (2010). Informe sobre tendencias sociales y educativas en América Latina. Metas educativas 2021: desafíos y oportunidades. Buenos Aires:IPE-UNESCO-OEI.

(2018). Base de datos. Recuperado 20/04/2018 de http://www.siteal.iipe.unesco.org/base_de_datos/consulta.

Steiner-Khamsi, G. (2010). The Politics and Economics of Comparison. Comparative Education Review 54 (3),323-342.

Tedesco, J. C. (2012). Educación y justicia social en América Latina. Buenos Aires: Fondo de Cultura Económica de Argentina. 
Vila Lladosa, L. E. (2003). Los beneficios no monetarios de la educación. Revista de Educación, 331, 309-324.

Urquiola, M. (2011). Calidad y cantidad educativa en Bolivia: 1996-2010. Informe preparado para el Ministerio de Relaciones Exteriores de Holanda. Recuperado 28/04/2018 de https://www.government.nl/documents/reports/2011/08/01/ iob-subrapport-urquiola-calidad-ycantidad-educativa-en-bolivia-1996-201. 